\title{
The Successful Treatment of Chronic Pain Using Microcurrent Point Stimulation Applied to Scars
}

\section{Abstract}

Objectives: Although microcurrent is widely used for chronic pain and stress management, as well as scar or neural therapy as a popular approach for the treatment of pain, there remains considerable controversy as to their combined therapeutic value in chronic pain management. We aimed to determine the effect and magnitude that DC microcurrent therapy has when applied to physical scars and its effects on a wide variety of non-specific chronic pain syndromes.

Design: This was a cohort study analysis of treatment outcomes pre, post and 48-hour follow-up after Microcurrent Point Stimulation (MPS) was applied to physical scars on 51 patients with history of non-specific pain.

Interventions: An MPS Scar Release protocol was applied bi-laterally to physical scars. Evaluations entailed a baseline Visual Analogue Score (VAS) pain scale assessment, which was repeated after an electro-therapy treatment and 48 hours later. All 51 patients received one Microcurrent Point Stimulation Scar Release session.

Outcome Measures: The VAS response of the 51 patient sample with chronic pain reflected a statistically significant reduction of 3.706 points or $59 \%$ reduction in mean pain levels post MPS Scar Release application, when compared to initial pain levels [95\% CI $(3.033,4.379 ; \mathrm{p}=0.0001]$. When VAS was measured at 48-hour follow-up, there was another statistically significant reduction of 0.902 points or $34 \%$ reduction in mean pain levels post treatment [95\% CI $(0.406,1.398$; $\mathrm{p}=0.001]$. Together, MPS Scar Release protocol produced a statistically significant reduction of 4.608 points or $73 \%$ reduction in mean pain levels post treatment, when compared to initial pain levels [ $95 \%$ CI $(3.940,5.275) ; \mathrm{p}=0.0001]$.

Conclusion: The positive results in this study could have applications to patients who have physical scars and are impacted by chronic pain syndromes.

Keywords: Scars; Microcurrent point stimulation; chronic pain
Research Article

Volume 10 Issue 3 - 2017

Gokal $\mathrm{R}^{1 *}$, Armstrong $\mathrm{K}^{2}$, Durant $\mathrm{J}^{3}$, Todorsky $\mathbf{W}^{4}$ and Miller $\mathbf{L}^{5}$

${ }^{1}$ University of Manchester, Manchester, United Kingdom

${ }^{2}$ Women's Integrative Healing, USA

${ }^{3}$ Brunswick, USA

${ }^{4} J a c k s o n v i l l e$, USA

${ }^{5}$ Advanced Pain Rehab, USA

*Corresponding author: Dr. Gokal R, University of Manchester, Consultant nephrologists, Royal infirmary, Manchester, UK, Email: ramgokal@rogers.com.

Received: December 12, 2017 | Published: December 28, 2017
Abbreviations : ANS: Autonomic Nervous System; AC: Alternating Current; DC: Direct current; VAS: Visual Analogue Scale

\section{Introduction}

Chronic pain affects millions of people every year and the effects of pain result in tremendous health care costs, in terms of rehabilitation and lost worker productivity, plus the emotional and financial burden it places on patients and their families. According to a recent Institute of Medicine Report: Relieving Pain in America: A Blueprint for Transforming Prevention, Care, Education, and Research, pain is a significant public health problem that costs society at least $\$ 560$ - $\$ 635$ billion annually, an amount equal to about $\$ 2,000$ for every living person in the U.S. This includes the total incremental cost of health care due to pain that ranges from $\$ 261-\$ 300$ billion to $\$ 297-\$ 336$ billion related to lost productivity (based on days of work missed, hours of work lost, and lower wages) $[1,2]$. In addition, there is currently a massive concern with the enormous use/abuse of analgesics and opioids throughout the USA [3-5]. If pain control can be achieved through other means as exemplified in this report, this could then impact favourably on this problem. Scars and trauma have long been recognized in neural therapy as a source of chronic pain as a result of Autonomic Nervous System (ANS) (in particular sympathetic nervous system) upregulation [6-10]. It is theorized that damaged local cells lose their normal membrane potential, transmitting abnormal electric signals throughout the rest of the body via the autonomic nervous system, acting as physical agonists to sympathetic upregulation (stress) and pain [11].

Traditionally, the modality of choice for electro-therapy has been Alternating Current (AC) [12-14]. However, there are two known types of electrical currents, AC and Direct current (DC). AC moves bi-directionally and is applied in the miliamperage range (10-3 amperes), usually called TENS or electro-acupuncture (EA) [15-17]. DC is uni-directional and when applied in the microamp or millionth of amp (10-6 -amperes) range to acupuncture or trigger points, is called Microcurrent Point stimulation (MPS) [18-19]. Microcurrent therapies involve applying weak direct currents $(80 \mu \mathrm{A}-1 \mathrm{~mA})$, and are now being increasingly recognized as an adjunct for pain relief and autonomic nervous system 
regulation [20-24]. It is theorized that electro-acupuncture and microcurrent electro-currents have different modulating affects on the autonomic nervous system and pain outcomes [25]. There is no consensus in the literature identifying the best practice measures for microcurrent applied to scars for the treatment of chronic pain. Although sufficient evidence supports the application of microcurrent and neural therapy for chronic pain, there is limited evidence in literature to support the application of electro-therapies to scars to reduce chronic pains. The purpose of this pilot study was to assess the impact of Microcurrent Point Stimulation Scar Release protocol applied to physical scars on the pain levels in a random sample of chronic pain patients, after single application.

\section{Patients, Materials and Methodology}

This study entailed the use of MPS in 51 patients ( 42 females, 9 males; mean age 47 years, SD 12.81) with chronic non-specific pains with a mean pain duration average of 7.61 years (SD 1.34) (Table 1) presenting to us for therapy of their problem. The location of the scars are shown in Table 2 and the pain location sites in Table 3. Inclusion criteria were simple: patients who were currently suffering from chronic pain for greater than 3 months, with a recorded $>4$ VAS Pain Scale score and have visible physical scar(s). Physical scars were defined as surgical or trauma induced. The diagnoses of pain, location, severity, sex, previous interventions or surgeries were not considered exclusion criteria. Informed consent was obtained to partake in treatment and the study assessments. Patient pain scores were recorded immediate pre treatment and twice post treatment: immediately after application, and again 48 hours later. Microcurrent Point Stimulation was simultaneously applied bi-laterally to scars using [26] two Dolphin Neurostim (Center for Pain \& Stress Research Ltd, Ontario, Canada) devices. This is an FDA-approved device which apply low frequency, concentrated, microcurrent stimulation for the relief of chronic pain and stress [24-25]. MPS application time was 30 seconds per point at approximate one-half inch intervals along the length of the scars. Polarity of application is important, as on one side of the scar, the device is set to negative pole $(-)$ and on the other side of scar, the second device is set to a positivenegative pole (+/-). The intent of this methodology is to push a negatively charged current back and forth through a positively charged (oriented) scar tissue. For the purpose of this study, only physical scars were treated, with the average treatment duration time of 30 minutes per patient.

Table 1: Descriptive statistics.

\begin{tabular}{|c|c|c|c|c|c|}
\hline \multicolumn{7}{|c|}{ Descriptive Statistics } \\
\hline & N & Minimum & Maximum & Mean & Std. Deviation \\
\hline Duration of Pain & 51 & 90 days & 36 years & 7.61 years & 1.339 years \\
\hline Age (years) & 51 & 21 & 72 & 47.34 & 12.812 \\
\hline Pain Before Treatment (0-10) & 51 & 4 & 10 & 6.33 & 1.912 \\
\hline Pain after Treatment (0-10) & 51 & 0 & 10 & 2.63 & 1.913 \\
\hline Follow up Pain - 2 Days after Treatment(0-10) & 51 & 0 & 10 & 1.73 & 1.930 \\
\hline
\end{tabular}

Table 2: Scar Location and percentages in the 51 patients.

\begin{tabular}{|c|c|c|}
\hline Scar Location & Total Number=51 & Percentage 100\% \\
\hline Abdomen & 31 & $60.70 \%$ \\
\hline Knee & 8 & $15.6 \%$ \\
\hline Hernia (abdomen) & 3 & $5.88 \%$ \\
\hline Ankle & 3 & $5.88 \%$ \\
\hline Breast & 2 & $3.9 \%$ \\
\hline Neck & 2 & $3.9 \%$ \\
\hline Wrist/Hand & 2 & $3.9 \%$ \\
\hline Total & 51 & $100 \%$ \\
\hline
\end{tabular}

Visual Analogue Scale (VAS) was used to evaluate the patient's pain. The VAS is an 11-point scale from $0-10$ with 0 being no pain and 10 being the most intense pain imaginable [27-30]. The patient verbally selects a value that is most in line with the intensity of the pain that they have experienced in the last 24 hours or is often reported as a rating during a specific movement pattern or functional task. The VAS has good sensitivity and excellent test-retest reliability [31].
Table 3: Pain Location in the 51 patients with percentages.

\begin{tabular}{|c|c|c|}
\hline Pain Location & Total Number=51 & Percentage 100\% \\
\hline Back & 20 & $39.2 \%$ \\
\hline Neck & 9 & $17.6 \%$ \\
\hline Shoulder & 8 & $15.6 \%$ \\
\hline Scapula (shoulder) & 1 & $1.96 \%$ \\
\hline Hip & 4 & $7.85 \%$ \\
\hline Finger/Hand & 3 & $5.88 \%$ \\
\hline Abdomen & 3 & $5.88 \%$ \\
\hline Arm & 1 & $1.96 \%$ \\
\hline Knee & 51 & $1.96 \%$ \\
\hline Total & & $100 \%$ \\
\hline
\end{tabular}

The aim of this cohort preliminary study was to evaluate whether

a) Microcurrent Point Stimulation Scar Release protocol, when applied to SCARS, can modulate or improve VAS pain scale in patients suffering with chronic pain. 
b) Microcurrent Point Stimulation Scar Release protocol, when applied to scars, is a valid option for the nonpharmacological pain management of chronic pain conditions.

\section{Results}

\section{Outcome Measures}

The VAS response of the 51 patient sample with chronic pain reflected a statistically significant reduction in mean post pain levels of 3.706 points or $59 \%$ reduction in mean pain levels post MPS application to physical scars, when compared to initial pain levels [95\% CI (3.033, 4.379; $\mathrm{p}=0.0001]$. When VAS was measured at the 48 hour follow-up, there was a further statistically significant reduction of 0.902 points or $34 \%$ reduction in mean pain levels post treatment [ $95 \%$ CI $(0.406,1.398 ; \mathrm{p}=0.001]$, for a total pain reduction of 4.608 points or $73 \%$ reduction in mean pain levels post MPS treatment, when compared to initial pain levels [95\% CI $(3.940,5.275) ; p=0.0001$ ] (Figure 1). There was no correlation between pain location and site of the physical scars (Tables $2 \& 3$ ).

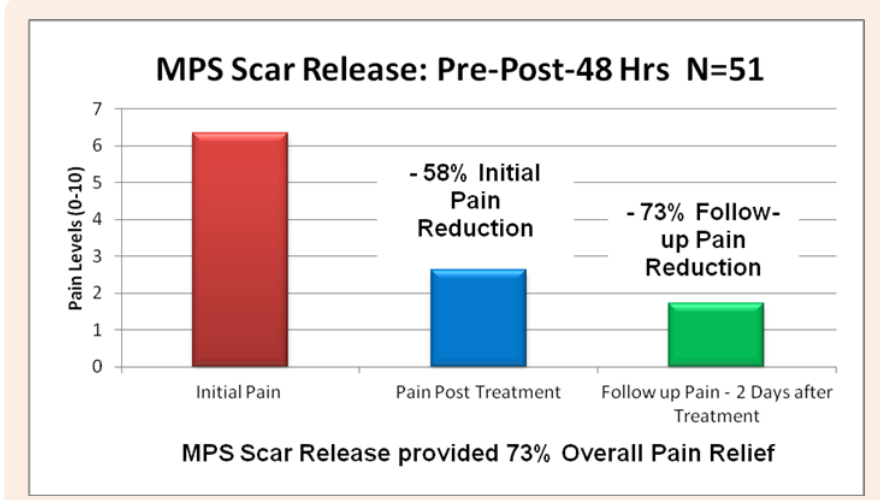

Figure 1: N=51 MPS-Scar Pain Graph.

\section{Discussion}

Chronic pain often equates to stress, both of which can make our daily lives miserable, and can lead to significantly impaired physical health and high societal costs $[1,2]$. For some time now, chronic pain has been difficult to diagnose and treat for many health care professionals. When the millions of physical scars produced annually throughout North America [32,33] are combined with the day-to-day accumulated patient traumas, the data represents a significant pre-existing pool of stress and pain patients within the general population $[32,33]$. It may help to explain the causation of symptoms for millions of chronic pain sufferers. In addition, the long-term use of opioids is now approaching epidemic levels in the USA, with few viable solutions for treatment in the foreseeable future [3-5]. Treatments like the kind described in this report could have a favourable impact on this problem.

The data from this study clearly shows that the application of Microcurrent Point Stimulation to physical scars had a marked improvement in pain outcomes when compared to baseline measurements in chronic pain patients. The improved outcomes were even more impressive given the patient sample for pain duration (mean 7.61 years) and the intensity (mean $6.33 / 10$ ) improved after a single MPS scar release application. Increased pain relief between post application and results 48 hours later was also noted as an improved outcome, suggesting internal functional changes may have occurred. It is suggested in the literature that DC microcurrent mimics human biocellular communications, enhancing autonomic nervous system regulation and the production of beta-endorphins, resulting in a body-wide therapeutic benefits [21,35]. These biochemical processes may provide a plausible explanation for the improved pain modulation over time after concentrated DC microcurrent is applied, and is an area where future research is required. We have previously reported, in several published studies, reduction in pain and cortisol with improvements in autonomic nervous system functionality in patients using MPS [18-21]. The consistency of chronic pain outcome improvements through the application of MPS to physical scars suggests there may be a strong relationship between chronic pain symptomology and physical scars throughout the body.

\section{Conclusion}

Chronic pain can limit quality of life, restrict work and social engagement, and is often blamed for the development of drug dependency of various forms. This study showed MPS Therapy applied to physical scars provided statistically significant reduction in initial pain levels with a further reduction after a 48-hour follow-up. These significant changes help validate the potential application of MPS to SCARS as an viable option to treating patients with non-specific soft tissue chronic pain. However, long term further investigation is warranted with a larger focus group to confirm these results and to assess their duration.

\section{Acknowledgements}

Statistical analyses were done by 3rd party freelance statistician using SPSS software, a widely used program for statistical analysis in social and medical science.

\section{Author Disclosure Statement}

All the authors whose names are listed in this study have a educational association with the sponsoring company that may create the appearance of a conflict of interest in connection with the submitted manuscript.

\section{References}

1. Relieving Pain in America. A Blueprint for Transforming Prevention, Care, Education, and Research. Institute of Medicine (US) Committee on Advancing Pain Research, Care, and Education. Washington (DC): National Academies Press (US); 2011. ISBN-13: 978-0-309-21484-1.

2. Gaskin DJ, Richard P (2012) The Economic Costs of Pain in the United States. The Journal of Pain 13(8): 715-724.

3. Han B, Compton WM, Blanco C, Crane E, Lee J, et al. (2017) Prescription Opioid Use, Misuse, and Use Disorders in U.S. Adults: 2015 National Survey on Drug Use and Health. Ann Intern Med 167(5): 293-301.

4. Muhuri PK, Gfroerer JC, Davies MC (2013) (CBHSQ [Center for Behavioral Health Statistics and Quality] Data Review, 2013). 
5. Carlson RG, Nahhas RW, Martins SS, Daniulaityte R (2016) Predictors of transition to heroin use among initially non-opioid dependent illicit pharmaceutical opioid users: A natural history study. Drug Alcohol Depend 160: 127-134.

6. Kidd RF (2005) Interference fields. In: Neural Therapy. Renfrew, Ontario, Custom Printers of Renfrew Ltd, Canada, pp. 24-40.

7. Williams L (2007) Blocks to healing: Chronic dominant foci. In: Radical Medicine: Cutting-Edge Natural Therapies That Treat the Root Causes of Disease. VT: Healing Arts Press, USA, pp. 402-420.

8. Brook M (2013) The Importance of Scar Tissue Release Therapy. Massage Today $9(6)$.

9. Baker R, Urso-Baiarda F, Linge C, Grobbelaar A (2009) Cutaneous scarring: A clinical review. Dermatol Res Pract 625376.

10. Defalque RJ (1982) Painful trigger points in physical scars. Anesth Analg 61(6): 518-520.

11. Dosch P (1984) Manual of Neural Therapy According to Huneke (11 edn), Stuttgart, Haug Publishers, Germany.

12. Andrew RJ, Mackler LS (2007-09-01). Clinical Electrophysiology: Electrotherapy and Electrophysiologic Testing ( $3^{\text {rd }}$ edn), In: Lippincott Williams \& Wilkins (Eds.), ISBN, 0781744849.

13. DeSantana JM, Walsh DM, Vance C, Rakel BA, Sluka KA (2008) Effectiveness of Transcutaneous Electrical Nerve Stimulation for Treatment of Hyperalgesia and Pain. Curr Rheumatol Rep 10(6): 492-499.

14. Johnson M, Martinson M (2007) "Efficacy of electrical nerve stimulation for chronic musculoskeletal pain: A meta-analysis of randomized controlled trials. Pain 130 (1-2): 157-165.

15. Nnoaham KE, Kumbang J (2008) Transcutaneous electrical nerve stimulation (TENS) for chronic pain. The Cochrane Library (3): CD003222.

16. Acupuncture Today: Electroacupuncture. 2004-02-01. Retrieved 2006-08-09.

17. Ezzo JM, Richardson MA, Vickers A, Allen C, Dibble SL, et al. (2006) Acupuncture-point stimulation for chemotherapy-induced nausea or vomiting". Cochrane Database Syst Rev (2): CD002285.

18. Chevalier A, Armstrong K, Gokal R (2016) Microcurrent Point Stimulation Applied to Acupuncture Points for the Treatment of NonSpecific Lower Back Pain. J Altern Complement Integr Med 23(4): 295-299.

19. Armstrong K, Gokal R, Chevalier A, Todorsky W, M Lim (2017) Microcurrent Point Stimulation Applied to Lower Back Acupuncture Points for the Treatment of Nonspecific Neck Pain 23(24): 1-5.

20. Chevalier A, Armstrong K, C Norwood-Williams, Gokal R (2016) DC Electroacupuncture Effects on Scars and Sutures of a Patient with Postconcussion Pain. Medical Acupuncture, Vol. 28(4): 223-229.
21. Armstrong K, Gokal R, Durant J, Todorsky W, Chevalier A, et al. (2017) Detailed Autonomic Nervous System Analysis of Microcurrent Point Stimulation Applied to Battlefield Acupuncture Protocol. Med Acupunct 29(2): 87-93.

22. McMakin C (2004) Microcurrent therapy: a novel treatment method for chronic low back myofascial pain. Journal of Bodywork and Movement Therapies 8(2):143-153.

23. Cheng N, Van Hoof H, Bockx E, Hoogmartens MJ, Mulier JC, et al. The effects of electric currents on ATP generation, protein synthesis, and membrane transport in Rat Skin. Clinical Orthopaedics and Related Research (171): 264-272.

24. Armstrong K (2016) Electro-Therapy Exposed.

25. Chevalier A, Armstrong K, Gokal R (2017) Detailed Heart Rate Variability, Exercise Tolerance, Cortical and Vas Pain Scale Analysis of Two Forms of Electro-Therapy Applied To A Patient with Chronic Back Neuropathic Pain. J Cell Mol Biol, UK.

26. http://www.accessdata.fda.gov/cdrh_docs/pdf13/K133789.pdf

27. Krebs EE, Carey TS, Weinberger M (2007) Accuracy of the Pain Numeric Rating Scale as a Screening Test in Primary Care. J Gen Intern Med 22(10): 1453-1458.

28. Williamson A, Hoggart B (2005) Pain: a review of three commonly used pain rating scales. J Clin Nurs 14(7): 798-804.

29. Jensen M, McFarland C (1993) Increasing the reliability and validity of pain intensity measurement in chronic pain patients. Pain 55(2): 195-203.

30. Hawker GA1, Mian S, Kendzerska T, French M (2011)Measures of Adult Pain Visual Analog Scale for Pain (VAS Pain): Numeric Rating Scale for Pain (NRS Pain): McGill Pain Questionnaire (MPQ): ShortForm McGill Pain Questionnaire (SF-MPQ): Chronic Pain Grade Scale (CPGS): Short Form-36 Bodily Pain Scale (SF-36 BPS): and Measure of Intermittent and Constant Osteoarthritis Pain (ICOAP). Arthritis Care Res 63(11): 240 -252.

31. Maniadakis N, Gray A (1999) The economic burden of back pain in the UK. Global Health Outcomes, Searle Division of Monsanto, UK, p. 95-103.

32. Agency for Healthcare Research and Quality. Overview of the National Inpatient Sample (NIS). Healthcare Cost and Utilization Project (HCUP). Rockville, MD: Agency for Healthcare Research and Quality, USA.

33. American Academy of Facial Plastic and Reconstructive Surgery. 310 South Henry Street, Alexandria, VA 22314. 703) pp. 6299-9291.

34. Mc Farlane A (2010) The long-term costs of traumatic stress: intertwined physicall and psychological consequence. World Psychiatry 9(1): 3-10.

35. Chang R, Pomeranz B (1979) Electroacupuncture analgesia could be mediated by at least two pain-relieving mechanisms: endorphin and non-endorphin systems. Life Sci 25(23): 1957-1962. 